\title{
Contra-Directional Coupling into Slotted Photonic Crystals for Spectrometric Applications
}

\author{
Mark G. Scullion, ${ }^{1 *}$ Andrea Di Falco, ${ }^{2}$ Thomas F. Krauss, ${ }^{1}$ \\ ${ }^{1}$ Department of Physics, University of York, York, UK \\ ${ }^{2}$ School of Physics and Astronomy, University of St Andrews, St Andrews, UK \\ *Corresponding author: mark.scullion@york.ac.uk
}

Received Month X, XXXX; revised Month X, XXXX; accepted Month X, XXXX; posted Month X, XXXX (Doc. ID XXXXX); published Month X, XXXX

\begin{abstract}
We propose and demonstrate the concept of a contra-directional coupler between a W1 and a slotted photonic crystal waveguide. The bandwidth and operating wavelength of such a coupler can be controlled via its geometrical parameters, and power transfer is not periodic unlike in the more familiar co-directional case. Light of specific wavelengths can be extracted from the W1 mode into air slot modes using this design, with W1/slot coupling efficiencies of up to $99 \pm 1 \%$, and waveguide extracted coupling efficiencies of up to $51 \pm 12 \%$ demonstrated experimentally. Combining several of these couplers in series, we demonstrate the spectral filtering functionality on-chip. The device therefore combines the well-known sensing function of the slotted waveguide geometry with the spectrometer function, thus uniting two essential biosensor functions in a monolithic device. (c) 2014 Optical Society of America
\end{abstract}

Slotted photonic crystals (SPhCs) [1-5] have recently demonstrated promise in a number of applications, such as biosensing [6-8], gas detection [9, 10] and optical modulation $[11,12]$ due to the strong confinement of light within the contents of the $\sim 100 \mathrm{~nm}$ scale air slot. This confinement gives greater light interaction with the slot content than a standard photonic crystal does, thus leading to a stronger dependence of the $\mathrm{SPhC}$ spectrum on the refractive index of its contents. The dispersive properties of slotted waveguides can, however, be very different from those of the more familiar W1 type, making coupling difficult. Several solutions $[11,13,14]$ have been proposed as in-line couplers for $\mathrm{SPhCs}$, each with different levels of efficiency and bandwidth. The side-coupled geometry has only been recently explored for coupling into slotted waveguides [15]. We here show a new W1 side coupled mechanism that can not only be used for coupling into $\mathrm{SPhCs}$, but can also be easily tuned to give wavelength and bandwidth selectivity, which is advantageous for filtering applications. The contradirectional nature of this coupling mechanism also gives potential for combining the spectrometric and sensing functions in a single device for Lab-on-a-chip applications.

The fundamental modes of a $\mathrm{SPhC}$ has a dispersion curve with gradient of opposite sign to that of a standard W1 as shown in Fig 1. The mode profile of a slotted waveguide also differs greatly from $\mathrm{W} 1$ and strip waveguides, thus matching of the modes requires a suitable coupler. We have previously used a coupler based on enlarged defects at the edge of the photonic crystal lattice and demonstrated its high efficiency and large bandwidth [14].

An alternative solution is to place the $\mathrm{SPhC}$ and the $\mathrm{W} 1$ in a side-coupled geometry. In this case the W1 and SPhC defects are considered within the same crystal lattice, but separated by several rows of holes. The corresponding bandstructures were computed using the MPB frequency domain package [16], and are shown in Fig 2 , for a crystal of effective index 2.7 , radius $0.294 a$ and slot width $0.27 a$,
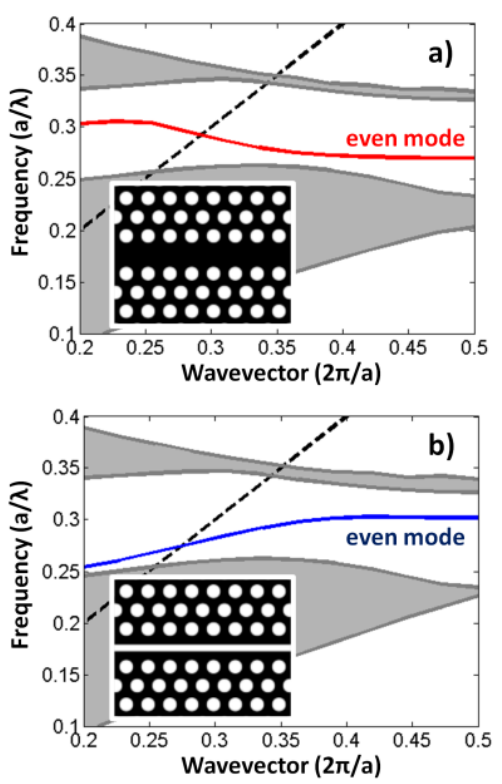

Fig. 1. Bandstructure and lattice geometry (inset) of (a) W1 defect photonic crystal. (b) Slotted defect photonic crystal. Dashed line indicates light line

where $a$ is the lattice constant. Here the close proximity of the $\mathrm{SPhC}$ and $\mathrm{W} 1$ results in an anti-crossing point, and hence a mini stopband being formed between the two fundamental modes of each defect. The bandwidth and frequency of this mini stopband can be controlled by simply changing the geometry of the lattice. A wider stop band results from placing the two defects closer together, whilst the frequency of the stopband is controlled by the width of the slot or the radius/position of the holes. The difference in gradient of the $\mathrm{W} 1$ and $\mathrm{SPhC}$ modes therefore results in light propagating in one structure to be back reflected into the other at the anti-crossing point, or in other words, to be contra-directionally coupled. 

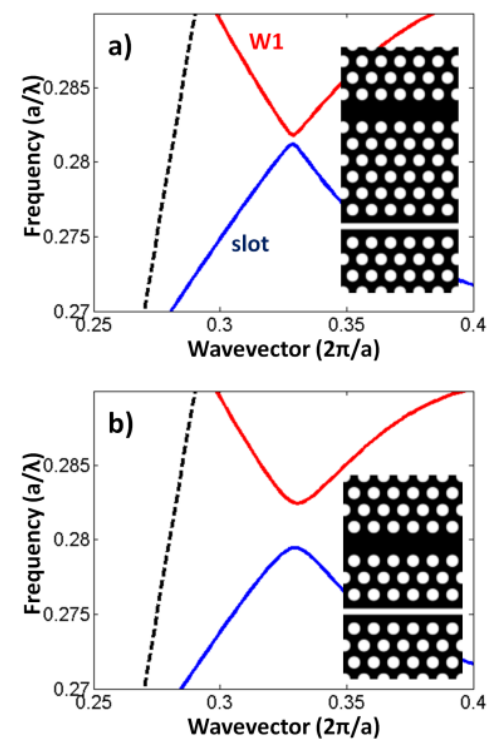

Fig. 2. Anti-crossing point created in bandstructure between SPhC and W1 separated by a spacer of (a) 6 rows of holes, (b) 3 rows of holes. Units normalized to period $a$.

a)
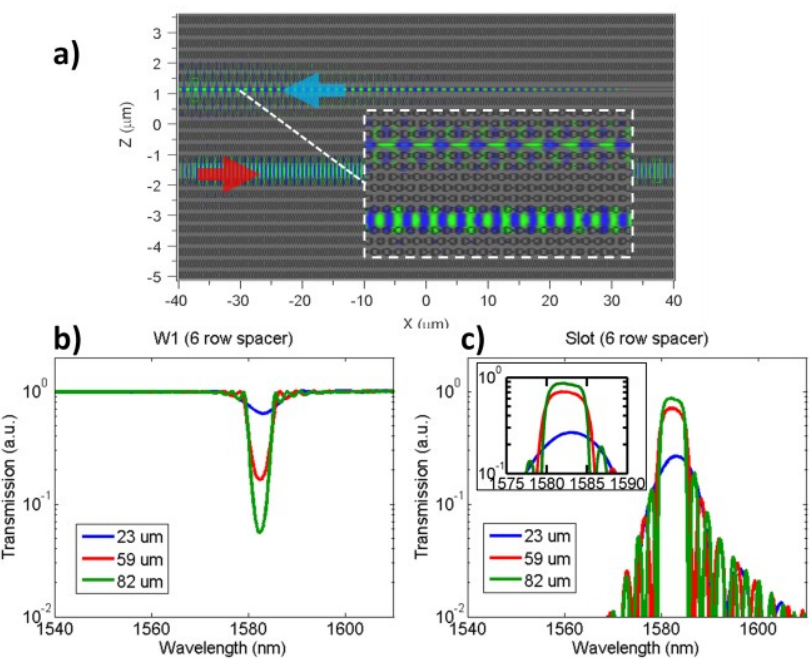

Fig. 3. a) Field profile of Ez component at a coupled wavelength and b) Simulated transmission spectrum of W1 and c) Slot channels of coupler of different lengths.

We studied the transmission properties of this structure with 2D Finite Difference Time Domain (FDTD) simulations. The period of the photonic crystal was chosen to be $440 \mathrm{~nm}$ and the structure length was varied
(23, 59 and $82 \mu \mathrm{m})$, whilst the other parameters were the same as the MPB simulations mentioned above. Fig 3a) shows the field profile of light coupled between the two defects for a spacer of 6 rows of holes. The corresponding $\mathrm{W} 1$ throughput, and the light dropped into the SPhC, are shown in Fig 3 b) and c). As expected from coupled mode theory [17], we find that structures with greater SPhC/W1 separation have narrower bandwidth, but require longer interaction length to transfer the same level of power. Simulations predict that an 82 micron long slot with a spacer of 6 rows of holes has a coupling efficiency of up to $87 \%$ at the central wavelength of the filter, and a

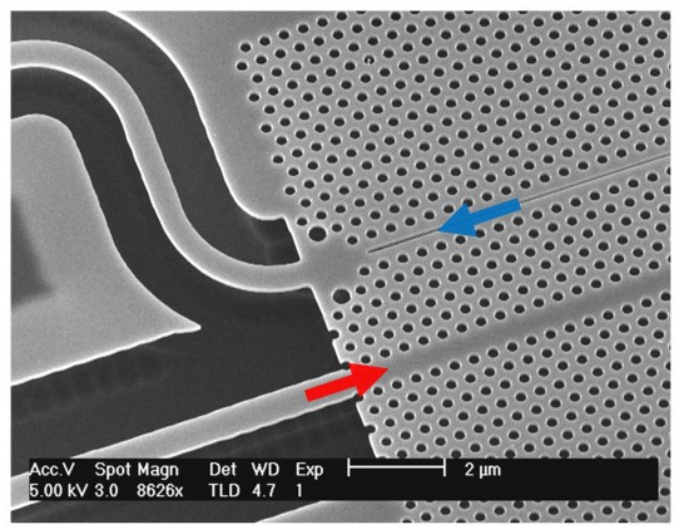

Fig. 4. SEM image of a fabricated structure.

bandwidth of $6 \mathrm{~nm}$ (Full Width at Half Maximum, FWHM) as shown in Fig 3. Coupling efficiencies are calculated relative to the transmission of the W1 waveguide feeding the slot. The main differences with $\mathrm{co}^{-}$ directional couplers is that light decays exponentially from one waveguide into the other, rather than being periodically exchanged [17], and couples into the backwards propagating mode of the output waveguide. The length of the coupling region is therefore less critical than a co-directional coupler; increasing the length just extracts more of the light. Alternatively, the separation distance can be reduced, which increases the bandwidth and reduces the required interaction length. Whilst contra-directional coupling has already been shown between photonic crystal waveguides [18, 19], and between waveguides and photonic crystals [20, 21], we show here for the first time (to our knowledge) the contradirectional coupling between a W1 and a slotted photonic crystal waveguide. The other advantage of this approach is that the light extraction can be made wavelengthspecific. This specificity not only allows light to be transferred between different media (from silicon into the slot), but the strong light matter interaction in the slotted architecture is also particularly attractive for sensing applications. 
To test these designs experimentally, we fabricated structures in Silicon-on-Insulator substrates $(220 \mathrm{~nm}$ top layer thickness) using electron beam lithography, reactive ion etching (RIE) and hydrofluoric acid (HF) undercutting to membrane the $\mathrm{SPhC}$ as previously described in [7, 14]. The geometrical parameters were chosen according to the simulated values. To measure transmission, the crystals
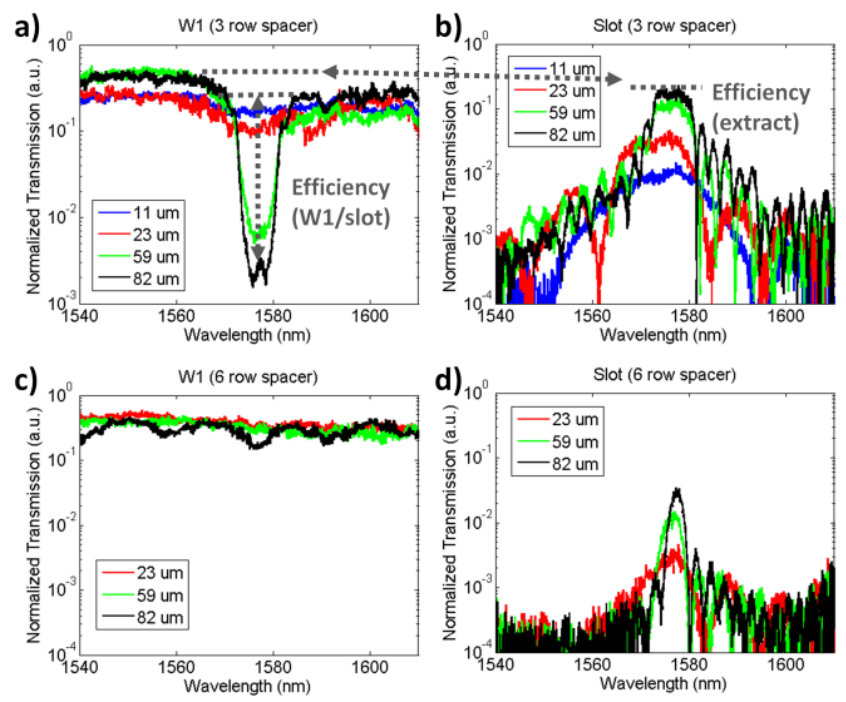

Fig. 5. a) Measured transmission spectrum from 3 row spacer a) W1 through b) slot drop. 6 row spacer c) W1 d) slot.

were mounted into an end-fire setup, with light being provided from an ASE (1520-1620 nm, $50 \mathrm{~mW}$ ), and sent via single mode fibres and lenses into and out of the chip. The spectrum was then measured using an optical spectral analyser, and normalized to a standard optical waveguide to remove the shape of the ASE. The interface

Table 1: Measured Coupling Efficiencies
\begin{tabular}{|c|c|c|c|c|}
\hline $\begin{array}{c}\text { W1/SPhC } \\
\text { Spacer }\end{array}$ & Length $(\mu \mathrm{m})$ & $\begin{array}{c}\text { FWHM } \\
\text { Bandwidth } \\
(\mathrm{nm})\end{array}$ & $\begin{array}{c}\text { Central } \\
\text { W1/Slot } \\
\text { Coupling } \\
\text { Efficiency (\%) }\end{array}$ & $\begin{array}{c}\text { Central } \\
\text { Extracted } \\
\text { Coupling } \\
\text { Efficiency (\%) }\end{array}$ \\
\hline 3 rows & 11 & 18 & $25 \pm 10$ & $5 \pm 1$ \\
\hline 3 rows & 23 & 14 & $52 \pm 10$ & $15 \pm 5$ \\
\hline 3 rows & 59 & 9 & $97 \pm 1$ & $45 \pm 20$ \\
\hline 3 rows & 82 & 9 & $99 \pm 1$ & $51 \pm 12$ \\
\hline 6 rows & 23 & 7 & - & $0.9 \pm 0.2$ \\
\hline 6 rows & 59 & 4 & - & $4 \pm 1$ \\
\hline 6 rows & 82 & 3 & $50 \pm 10$ & $9 \pm 3$ \\
\hline
\end{tabular}

described in [14] was used to couple light from the slot mode into a waveguide that looped around the crystal before terminating at the back facet of the chip, as can be inferred from the bottom of Fig. 4a. An SEM image of a device and the measured transmission spectra are shown in Fig 4. Photonic crystals of the same W1/SPhC geometry but of different length and spacers were also fabricated and measured. The results are shown in Figs 5, 6 and in

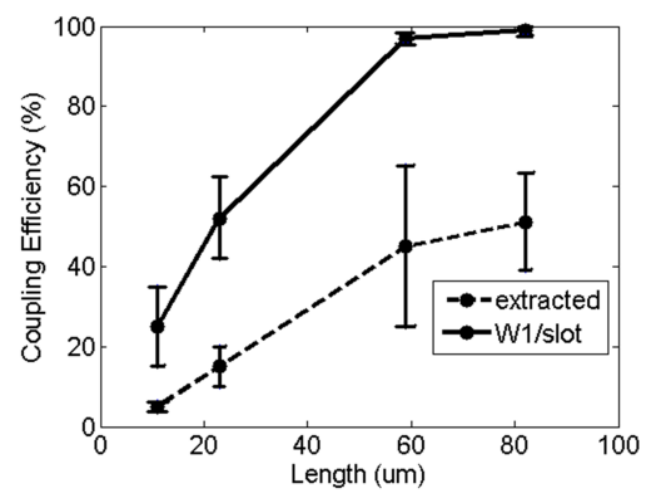

Fig. 6. Measured coupling efficiencies versus length for 3 row spacer coupler.

Table 1. Coupling efficiencies are calculated at the central wavelength of the coupled light, relative to the W1 transmission. Two coupling efficiencies can be inferred. The first we call the W1/slot coupling efficiency which is determined by comparing the dip in the W1 spectrum with the baseline transmission as depicted by the dashed lines in Fig 5a). This is the light coupled from the W1 into

a)

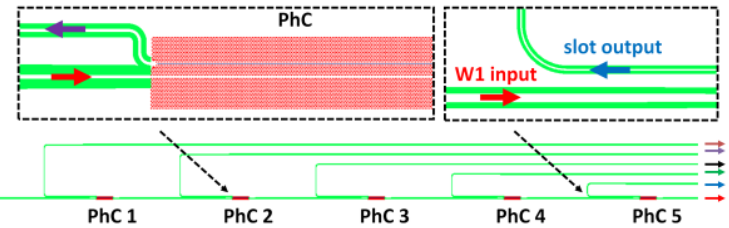

b)
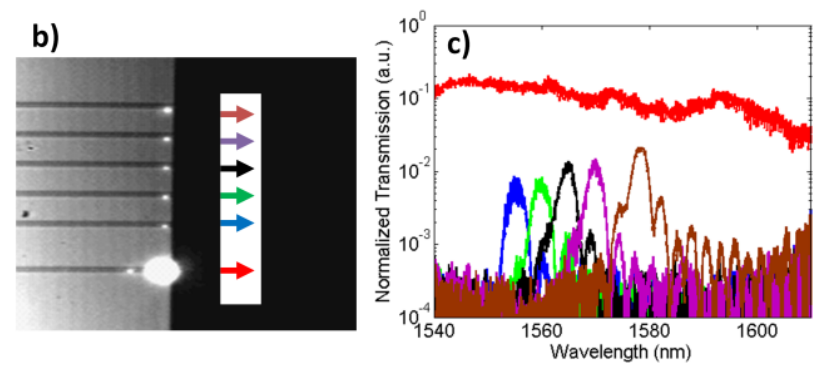

Fig. 7. a) Schematic of five filter design b) Image of output at back facet c) Measured transmission spectrum from each channel.

the $\mathrm{SPhC}$. The second efficiency we term the extracted efficiency which is the ratio of the peak of the slot drop spectrum to the base level of the W1 as shown in Fig 5 b). This efficiency corresponds to the amount of light actually measured from the waveguide extracting light from the slot channel. This efficiency is lower due to losses between the $\mathrm{SPhC}$ and waveguide. The level of coupling between the $\mathrm{W} 1$ and $\mathrm{SPhC}$ is lower than that predicted by simulation $(50 \pm 10 \% \mathrm{~W} 1 / \mathrm{SPhC}$ efficiency, $9 \pm 3 \%$ extracted efficiency experimentally and $87 \% \mathrm{~W} 1 / \mathrm{SPhC}$ simulation for a spacer of 6 rows of holes). This difference can partly be explained by: losses in the loops and slot/waveguide interface, propagation losses, fabrication errors, and the fact that the simulation is only $2 \mathrm{D}$. There is however a remaining discrepancy that should be the subject of future study. 
Next, we used the wavelength and bandwidth dependence to implement a filter function. Our design consisted of five such W1/SPhC couplers placed in series, with each SPhC having a slightly different slot width in order to shift the slot mode's dispersion curve, thereby altering the spectral position, and hence the central wavelength, of the anti-crossing point. Each device therefore filters out a different wavelength from the W1 bus. Since the waveguide separation remains constant, the bandwidth of each channel is identical. In the resulting five-filter design, shown in Fig 6, each slot therefore extracts a different wavelength of light into its own separate channel. The spectra are designed to overlap, as this allows finer resolution and higher densities of channels to be realised. Greater power transfer could also be realised by making the waveguides longer. Narrower line width could be achieved by increasing the $\mathrm{W} 1 / \mathrm{SPhC}$ separation, but this necessitates making the structure even longer. The strong interaction of the slot mode with the surrounding environment would make this design interesting for compact on-chip gas absorption detection, as the device could double as a sensor and spectrometer. Several channels could be designed to match known gas lines, whilst others could be used as a reference. As most gases absorb at Mid-IR wavelengths, this requires scaling the device up [22], whilst the silica in SOI absorbs at Mid-IR wavelengths, necessitating the use of different substrate layers such as sapphire or the use of undercut strip waveguides [23]. Assuming an absorption coefficient of $80 \mathrm{~cm}^{-1} \mathrm{CO}_{2}$ gas at the absorption lines around $4 \mu \mathrm{m}$ [24], free space propagation of light over a distance of $200 \mu \mathrm{m}$ in $400 \mathrm{ppm}$ (parts per million) gas would result in absorption of approximately $0.1 \%$. Utilizing slow light effects in slotted photonic crystals [2] may allow this number to approach 1 $\%$, making it a viable sensor for atmospheric gases. Other applications with higher gas concentrations would of course have better performance. All of these issues will be the subject of future study.

In summary, we have demonstrated that light can be coupled between W1 and slotted photonic crystals contradirectionally, and that the frequency and bandwidth of this coupling can be controlled via the geometric parameters. As SPhCs can have a free spectral range of more than $200 \mathrm{~nm}$, can operate at almost any nonabsorbing wavelength in silicon through scaling of the period, and that the bandwidth can be varied as shown in Table 1, then they can be useful for on-chip spectrometer functions. The added benefit of the strong light-matter interaction in the slot mode makes the design particularly attractive for dual use as a sensor and spectrometer.

\section{Acknowledgements}

We acknowledge support by the EPSRC of the UK, grant No. EP/J01771X/1 "Structured Light" for financial support. ADF acknowledges support from EPSRC (EP/I004602/1 and EP/J004200/1).

\section{References.}

1. A. Di Falco, L. O'Faolain, and T.F. Krauss, Photon. Nano. Fund. Appl. 6, 38 (2008).
2. A. Di Falco, L. O'Faolain and T.F. Krauss, Appl. Phys. Lett. 92, 083501 (2008).

3. T. Yamamoto, M. Notomi, H. Taniyama, E. Kuramochi, Y. Yoshikawa, Y. Torii, and T. Kuga, Opt. Exp. 16, 13809 (2008).

4. K. Foubert, L. Lalouat, B. Cluzel, E. Picard, D. Peyrade, F. de Fornel, and E. Hadji, Appl. Phys. Lett. 94, 251111 (2009).

5. C. Caer, X. Le Roux, V. K. Do, D. Marris-Morini, N. Izard, L. Vivien, D. Gao, and E. Cassan, IEE Photon. Tech. Lett. 23, 1298 (2011).

6. A. Di Falco, L. O'Faolain and T.F. Krauss, Appl. Phys. Lett. 94, 063503 (2009).

7. M. G. Scullion, A. Di Falco, and T.F. Krauss, Biosens. Bioelect. 27, 101 (2011).

8. S. Kita, S. Hachuda, S. Otsuka, T. Endo, Y. Imai, Y. Nishijima, H. Misawa, and T. Baba, Opt. Exp. 19, 17683 (2011).

9. W.C. Lai, S. Chakravarty, X. Wang, C. Lin, and R.T. Chen, Opt. Lett. 36, 984 (2011).

10. J. Jagerska, H. Zhang, Z. Diao, N. Le Thomas, and R. Houdre, Opt. Lett. 35, 2523 (2010).

11. J.M. Brosi, C. Koos, L.C. Andreani, M. Waldow, J. Leuthold, and W. Freude, Opt. Exp. 16, 4177 (2008).

12. J.H. Wulbern, J. Hampe, A. Petrov, M. Eich, J. Luo, A.K.Y. Jen, A. Di Falco, T.F. Krauss, and J. Bruns, Appl. Phys. Lett. 94, 241107 (2009).

13. X. Chen, W. Jiang, J. Chen, L. Gu, and R.T. Chen, Appl. Phys. Lett. 91, 091111 (2007).

14. M.G. Scullion, T.F. Krauss, and A. Di Falco, IEEE Photon. J. 3, 203 (2011).

15. R. Palmer, L. Alloatti, D. Korn, W. Heni, P. C. Schindler, J. Bolten, M. Karl, M. Waldow, T. Wahlbringk, W. Freude, C. Koss and J. Leuthold, IEEE Photon. J. 5, 2200409, (2012).

16. S.G. Johnson and J.D. Joannopoulos, Opt. Exp. 8, 173 (2001).

17. A. Yariv, IEEE J. Quant. Elect. 9, 919 (1973).

18. X.Y. Mao, D.B. Yao, L.Y. Zhao, Y.D. Huang, W. Zhang, and J.D. Peng, Chin. Phys. Lett. 25, 141 (2008).

19. M. Qiu, and M. Swillo, Photon. Nano. Fund. Appl. 1,23 (2003).

20. M. Grande, L. O'Faolain, T.P. White, M. Spurny, A. D’Orazio, and T.F. Krauss, Opt. Lett. 34, 3292 (2009).

21. W. Shi, X. Wang, W. Zhang, L. Chrostowski, and N.A.F. Jaeger, Opt. Lett. 36, 3999 (2011).

22. C. Reimer, M. Nedeljkovic, D.J.M. Stothard, M. O.S. Esnault, C. Reardon, L. O'Faolain, M. Dunn, G.Z. Mashanovich, and T.F. Krauss, Opt. Exp. 20, 29361 (2012).

23. Y. Xia, C. Qiu, X. Zhang, W. Gao, J. Shu and Q. Xu, Opt. Lett. 38, 1122 (2013).

24. J. Hodgkinson and R.P. Tatam, Meas. Sci. Tech. 24, 012004 (2013). 


\section{Full References.}

1. A. Di Falco, L. O'Faolain, and T.F. Krauss, "Photonic crystal slotted slab waveguides", Photonics and Nanostructures - Fundamentals and Applications 6, 38 (2008).

2. A. Di Falco, L. O'Faolain and T.F. Krauss, "Dispersion control and slow light in slotted photonic crystal waveguides", Applied Physics Letters 92, 083501 (2008).

3. T. Yamamoto, M. Notomi, H. Taniyama, E. Kuramochi, Y. Yoshikawa, Y. Torii, and T. Kuga, "Design of a high-Q air-slot cavity based on a width-modulated line-defect in a photonic crystal slab", Optics Express 16, 13809 (2008).

4. K. Foubert, L. Lalouat, B. Cluzel, E. Picard, D. Peyrade, F. de Fornel, and E. Hadji, "An airslotted nanoresonator relying on coupled high Q small V Fabry-Perot nanocavities", Applied Physics Letters 94, 251111 (2009).

5. C. Caer, X. Le Roux, V. K. Do, D. Marris-Morini, N. Izard, L. Vivien, D. Gao, and E. Cassan, "Dispersion Engineering of Wide Slot Photonic Crystal Waveguides by Bragg-Like Corrugation of the Slot", IEE Photonics Technology Letters 23, 1298 (2011).

6. A. Di Falco, L. O'Faolain and T.F. Krauss, "Chemical sensing in slotted photonic crystal heterostructure cavities", Applied Physics Letters 94, 063503 (2009).

7. M. G. Scullion, A. Di Falco, and T.F. Krauss, "Slotted photonic crystal cavities with integrated microfluidics for biosensing applications", Biosensors and Bioelectronics 27, 101 (2011).

8. S. Kita, S. Hachuda, S. Otsuka, T. Endo, Y. Imai, Y. Nishijima, H. Misawa, and T. Baba, "Supersensitivity in label-free protein sensing using a nanoslot nanolaser", Optics Express 19, 17683 (2011).

9. W.C. Lai, S. Chakravarty, X. Wang, C. Lin, and R.T. Chen, "On-chip methane sensing by near-IR absorption signatures in a photonic crystal slot waveguide", Optics Letters 36, 984 (2011).

10. J. Jagerska, H. Zhang, Z. Diao, N. Le Thomas, and R. Houdre, "Refractive index sensing with an air-slot photonic crystal nanocavity", Optics Letters 35, 2523 (2010).

11. J.M. Brosi, C. Koos, L.C. Andreani, M. Waldow, J. Leuthold, and W. Freude, "High-speed low" voltage electro-optic modulator with a polymerinfiltrated silicon photonic crystal waveguide", Optics Express 16, 4177 (2008).

12. J.H. Wulbern, J. Hampe, A. Petrov, M. Eich, J. Luo, A.K.Y. Jen, A. Di Falco, T.F. Krauss, and J. Bruns, "Electro-optic modulation in slotted resonant photonic crystal heterostructures", Applied Physics Letters 94, 241107 (2009).

13. X. Chen, W. Jiang, J. Chen, L. Gu, and R.T. Chen, " $20 \mathrm{~dB}$-enhanced coupling to slot photonic crystal waveguide using multimode interference coupler", Applied Physics Letters 91, 091111 (2007).
14. M.G. Scullion, T.F. Krauss, and A. Di Falco, "High Efficiency Interface for Coupling Into Slotted Photonic Crystal Waveguides", IEEE Photonics Journal 3, 203 (2011).

15. R. Palmer, L. Alloatti, D. Korn, W. Heni, P. C. Schindler, J. Bolten, M. Karl, M. Waldow, T. Wahlbringk, W. Freude, C. Koss and J. Leuthold, "Low Loss Silicon Strip-to-Slot Mode Converters", IEEE Photonics Journal 5, 2200409, (2012).

16. S.G. Johnson and J.D. Joannopoulos, "Blockiterative frequency-domain methods for Mawxell's equations in a planewave basis", Optics Express 8, 173 (2001).

17. A. Yariv, "Coupled-Mode Theory for GuidedWave Optics", IEEE Journal of Quantum Electronics 9, 919 (1973).

18. X.Y. Mao, D.B. Yao, L.Y. Zhao, Y.D. Huang, W. Zhang, and J.D. Peng, "An Integrative Biosensor Based on Contra-Directional Coupling Twodimensional Photonic Crystal Waveguides", Chinese Physics Letters 25, 141 (2008).

19. M. Qiu, and M. Swillo, "Contra-directional coupling between two-dimensional photonic crystal waveguides", Photonics and Nanostructures - Fundamentals and Applications 1, 23 (2003).

20. M. Grande, L. O’Faolain, T.P. White, M. Spurny, A. D'Orazio, and T.F. Krauss, "Optical filter with very large stopband $(300 \mathrm{~nm})$ based on a photonic-crystal vertical-directional coupler", Optics Letters 34, 3292 (2009).

21. W. Shi, X. Wang, W. Zhang, L. Chrostowski, and N.A.F. Jaeger, "Contradirectional couplers in silicon-on-insulator rib waveguides", Optics Letters 36, 3999 (2011).

22. C. Reimer, M. Nedeljkovic, D.J.M. Stothard, M. O.S. Esnault, C. Reardon, L. O'Faolain, M. Dunn, G.Z. Mashanovich, and T.F. Krauss, "Midinfrared photonic crystal waveguides in silicon", Optics Express 20, 29361 (2012).

23. Y. Xia, C. Qiu, X. Zhang, W. Gao, J. Shu and Q. $\mathrm{Xu}$, "Suspended $\mathrm{Si}$ ring resonator for mid-IR application”, Optics Letters 38, 1122 (2013).

24. J. Hodgkinson and R.P. Tatam, "Optical gas sensing: a review", Measurement Science and Technology 24, 012004 (2013). 